\title{
Percepción de la capacitación a Dentistas Generales de Zona: encuesta nacional
}

\author{
RICARDO CARTES-VELÁSQUEZ ${ }^{(1)}$
}

\section{RESUMEN}

Introducción: Informalmente sabemos que existe una mala percepción de la capacitación y formación entregada por los servicios de salud (SS) a los Dentistas Generales de Zona (DGZ) por medio del Programa Anual de Capacitación (PAC). El objetivo de este trabajo es determinar los distintos aspectos de esta percepción. Material y Método: Se realizó un estudio descriptivo de corte transversal, vía encuesta electrónica, contestada por 152 DGZ. Resultados: Los resultados muestran que el 81\%, $82,3 \%$ y 58,6\% no consideran aceptables la información, oportunidades y calidad, respectivamente, de los cursos ofrecidos por los SS a través del PAC. El 25\% no recibe educación continua (EC) por parte de su SS, ninguno de los encuestados ha recibido un curso de Magíster, 40,1\% ha realizado pasantias en sus hospitales base y 79,6\% cree que su SS no se preocupa por capacitarlo. En temáticas de APS, la mayoría cree contar un nivel de habilidades al menos aceptable. El 52,6\% considera al DGZ como un camino de formación para el desarrollo integral de la profesión y el 36,8\% cree llegar en igualdad de condiciones (destrezas) que sus contrapartes autofinanciadas a los programas de especialidad. Discusión: Las respuestas abiertas refuerzan la mala percepción de la EC entregada por los SS, la priorización de los médicos en detrimento de los DGZ y la autogestión como única vía para obtener una EC de calidad. Los hallazgos de este estudio confirman una situación que necesita de intervenciones para mejorar el desarrollo profesional de los DGZ que redunde en una atención de calidad a los pacientes.

Palabras clave: Educación continua, dentista, Chile, atención primaria.

\section{ABSTRACT \\ PERCEPTION OF REGIONAL GENERAL DENTISTS TRAINING: NATIONAL SURVEY}

Introduction: Informally, we know that the training offered by health services to Regional General Dentists (DGZ, Dentistas Generales de Zona) through the Yearly Training Program (PAC, Programa Annual de Capacitacion) is poorly perceived. The goal of this study is to determine different aspects of this perception. Materials and Methods: A descriptive cross-sectional study was carried out via an online survey, answered by 152 DGZs. Results: $81 \%, 82.3 \%$, and $58.6 \%$, respectively, did not consider the information, opportunities, and quality of the courses offered by health services through the PAC to be acceptable. 25\% did not receive continuing education through health services, none of those surveyed had received a Master's level course, 40.1\% had done internships in their base hospitals, and 79.6\% believed that health services was not worried about training them. In Primary Health Care (APS, Atencion Primaria en Salud), the majority felt they had a skill level that was at least acceptable. 52.6\% considered the DGZ was a path for carreer development, and $36.8 \%$ believed that they arrived at specialty programs with the same skill set as their self-funded counterparts. Discussion: The open answers reinforced the poor perception of continuing education offered by health services, and self-management as they only way to achieve quality education. The findings of this study confirm that this situation needs intervention to be improve DGZ's professional development and resulting quality patient care.

Key words: Continuing education, dentist, Chile, primary health care.

(1) Cirujano dentista. Etapa de Destinación y Formación. Cesfam Ralco. Alto Biobío. Servicio de Salud Biobío.Villa Ralco s/n, Alto Biobío. ricardo@cartesvelasquez.com 


\section{INTRODUCCIÓN}

La educación continua (EC) es en la actualidad un deber para prácticamente todos los trabajadores, dado el continuo avance en el conocimiento y las tecnologías, que muchas veces dejan obsoleto lo aprendido durante la fase de formación de nuestras respectivas profesiones. Esto es particularmente cierto en el área biomédica, por cierto en la odontología ${ }^{1,}$ ${ }^{2}$, que bajo el concepto de medicina basada en la evidencia (MBE) nos obliga a un continuo cuestionamiento y desarrollo de nuevas formas diagnósticas, terapéuticas y pronósticas que entregamos a nuestros pacientes; ; así, entonces, para quienes día a día atienden pacientes, la EC es un imperativo (bio)ético que va más allá del desarrollo profesional y de realizar buenas prácticas.

En Chile,y en línea con lo anterior, los funcionarios del Estado cuentan con la capacitación como un derecho laboral, representando -además- una de las principales vías para mejorar sus condiciones salariales (ascenso de grado) a través de la carrera funcionaria ${ }^{4}$. En el sector salud el aseguramiento de este derecho se materializa a través de la existencia del Programa Anual de Capacitación (PAC) a nivel de los servicios de salud (SS) y de los comités de capacitación (CC) en los establecimientos de dependencia municipal. En el caso de los Dentistas Generales de Zona (DGZ) en Etapa de Destinación y Formación (EDF), como funcionarios dependientes de los $\mathrm{SS}^{5}$, la capacitación es coordinada mediante el PAC.

Informalmente sabemos que para la mayoría de los DGZ las oportunidades de capacitación son reducidas y existe un sentimiento de falta de preocupación por parte de los SS hacia el estamento odontológico en general, pero no contamos con un diagnóstico con cifras precisas que pueda orientar a la implementación de posibles soluciones al respecto.

El objetivo del presente trabajo es determinar cuál es la percepción de los DGZ con respecto a los distintos aspectos de la EC entregada por los SS y la vía de formación profesional que representa la EDF.

\section{MATERIAL Y METODO}

Se realizó un estudio descriptivo de corte transversal en el grupo objetivo de los DGZ, dado que no existen registros fiables de todos ellos. Se utilizó la base de datos del sitio web de la Agrupación Nacional DGZ-EDF, que incluía 267 perfiles, siendo la más fiable y extensa, considerando que aproximadamente existen 300 DGZ a lo largo de Chile.

La recolección de los datos se realizó mediante una encuesta electrónica diseñada en la plataforma LimeSurvey, enviada por email con 2 recordatorios cada 2 semanas. La encuesta, de carácter anónimo y voluntario, consistía en 30 preguntas cerradas y 2 abiertas agrupadas en 5 secciones:

1. Datos personales y laborales.

2. Información, oportunidades y calidad de la capacitación entregadas por el PAC del SS.

3. Oportunidades de capacitación y autodiagnóstico de capacidades en temáticas APS.

4. Valoración de la EDF como camino de formación hacia la especialización.

5. Percepción de la capacitación entregada y la que necesitan los DGZ.

Para las variables que evaluaron percepción se utilizó una escala nominal ordinal de 7 niveles: pésimo, deficiente, regular, aceptable, bueno, muy bueno y sobresaliente; el resto se manejaron como variables dicotómicas: SI / NO.

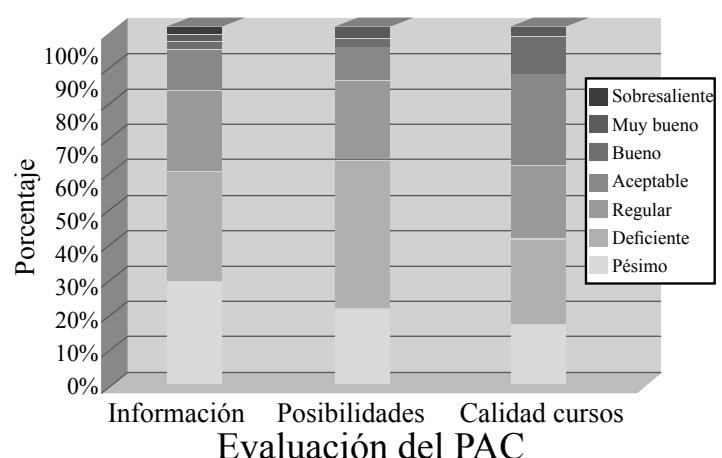

Figura 1. Distribución porcentual de la evaluación de la información, posibilidades y calidad de los cursos entregados por el PAC. 
Los datos fueron tabulados y graficados en el software MS Excel 2003 y analizados en Stata $10 /$ SE.

\section{RESULTADOS}

Tras 6 semanas online, la encuesta fue contestada por 172 DGZ, 152 en su totalidad para ser consideradas en el análisis, lo que representa una tasa de respuesta del $56,9 \%$. Al ser esta la primera investigación que aborda el tema no existen datos previos, pero si consideramos que en el mejor de los casos (apreciación del autor) un $25 \%$ de los DGZ tiene una buena percepción de la capacitación que reciben de sus SS, para un nivel de confianza del $95 \%$, los datos nos permitirán obtener una precisión absoluta del 4,5\% en los resultados.

De las encuestas completadas, 88 (57,9\%) corresponden a mujeres y $64(42,1 \%)$ a hombres; de los 29 SS del país, los de mayor representación corresponden a Coquimbo con 18; Nuble con 11; Araucanía Sur; Concepción; Osorno y Valdivia con 10; en cuanto al año de ingreso a la EDF los más representados fueron 2007 con 31, 2009 con 25 y 2010 con 26.

La Figura 1 muestra la percepción que tienen los DGZ del PAC: el 81\% considera "no aceptable" la información que éste entrega; con respecto a las posibilidades de acceder a capacitación el porcentaje alcanza un $82,3 \%$, y en la calidad de los cursos entregados es de $58,6 \%$. El 83,5\% de los encuestados no ha participado en la Comisión del PAC.

Un $25 \%$ de los DGZ no recibe capacitaciones desde el PAC; una por año, el 50,7\%; 2 por año, el $15,8 \%$ y el $8,5 \%, 3$ o más. Un $17,8 \%$ ha accedido a algún curso a nivel de diplomado o perfeccionamiento, ninguno de los DGZ encuestados ha cursado un magíster por medio del PAC. El 40,1\% ha participado de pasantías en sus hospitales de referencia. El 79,6\% cree que no existe una preocupación de los SS por capacitarlos de forma continua.

En la Tabla 1 podemos ver la proporción de DGZ que han tenido acceso a distintos cursos según su temática, las de mayor pertinencia para la práctica odontológica en APS.
Tabla 1. Distribución porcentual de la participación en capacitaciones ofrecidas por el PAC según temática.

\begin{tabular}{|l|l|l|}
\hline Temática & SI & NO \\
\hline Administración & $34,9 \%$ & $65,1 \%$ \\
\hline Atención preescolares & $13,8 \%$ & $86,2 \%$ \\
\hline Atención niños & $11,8 \%$ & $88,2 \%$ \\
\hline $\begin{array}{l}\text { Atención necesidades } \\
\text { especiales }\end{array}$ & $6,6 \%$ & $93,4 \%$ \\
\hline $\begin{array}{l}\text { Atención adultos mayo- } \\
\text { res }\end{array}$ & $6,6 \%$ & $93,4 \%$ \\
\hline $\begin{array}{l}\text { Manejo emergencias } \\
\text { médicas }\end{array}$ & $23 \%$ & $77 \%$ \\
\hline $\begin{array}{l}\text { Odontología Basada en } \\
\text { la Evidencia }\end{array}$ & $9,2 \%$ & $90,8 \%$ \\
\hline
\end{tabular}

La Figura 2 muestra el autodiagnóstico de capacidades para cada una de las temáticas mencionadas anteriormente, teniendo éstas niveles de, a lo menos, aceptable en: 59,2\% para administración; 78,9\% para la atención de preescolares; $98 \%$ para la de niños y adolescentes; $88,2 \%$ para adultos mayores; $48,7 \%$ para pacientes con necesidades especiales; $70,4 \%$ en el manejo de emergencias médicas durante la atención dental, y $36,8 \%$ en practicas basadas en la evidencia.

E1 52,6\% de los DGZ considera a la EDF como una vía aceptable de desarrollo profesional en el más amplio sentido de lo que significa ser cirujano dentista en el camino a convertirse en especialista. En términos de destreza clínica, un $36,8 \%$ cree llegar en igualdad de condiciones al programa de especialidad frente a sus pares autofinanciados. El 93,4\% considera ser DGZ como un camino válido para convertirse en especialista y en este sentido un $88,2 \%$ lo recomendaría a los recién titulados.

La primera de las preguntas abiertas, en relación a la percepción de la capacitación que se recibe del PAC, mostró una mayoría de respuestas:

- Quejándose de la poca información y posibilidades entregadas (especialmente a los que se desempeñan en establecimientos mu- 
nicipales).

- Una preocupación de los SS centrada en la capacitación de los médicos.

- La importancia de la autogestión para lograr capacitaciones de calidad.

La segunda pregunta fue enfocada a qué cursos creían necesarios se entregaran en el PAC, siendo mayoritariamente mencionados:

- Gestión

- Atención de preescolares

- Manejo de emergencias médicas

- Protección radiológica

- Ortodoncia interceptiva

- Pasantías en los hospitales base

\section{DISCUSION}

Siendo los DGZ un grupo bastante definido, en términos cuantitativos y cualitativos, es interesante observar los presentes resultados. En teoría debiesen tener un mejor acceso a capacitación que el resto del estamento odontológico debido a beneficios administrativos ${ }^{5} \mathrm{y}$ a una mayor necesidad de capacitarse producto de las postulaciones a las becas de especialidad, por tanto estos resultados representan a un sector privilegiado en este aspecto.

Es importante mencionar las limitaciones del estudio, que al ser inédito en esta área asume características de exploratorio: primero, la

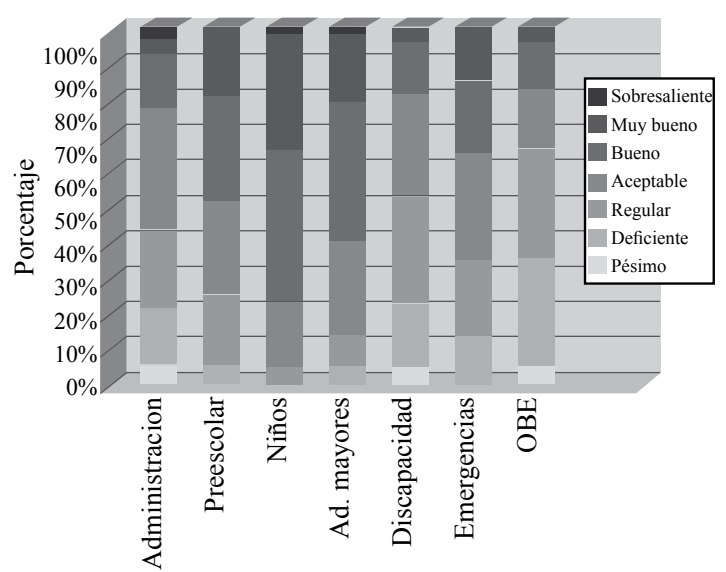

Figura 2. Distribución porcentual de las capacidades por temática según autodiagnóstico. cantidad de encuestados apenas sobrepasa la mitad del universo en estudio; segundo, la utilización de una base de datos no oficial (no gubernamental) ante la inexistencia de ella; tercero, la cantidad y orientación de las preguntas no abarca todos los aspectos de la capacitación de los dentistas, enfocándose a las áreas que el autor consideró de relevancia en la práctica clínica de la atención primaria.

Los resultados muestran una situación claramente preocupante: en promedio sólo 1 de cada 6 DGZ considera aceptable la información y las posibilidades de capacitación que les otorgan sus SS, cifra que se refuerza al preguntar si nota una preocupación de éste por capacitarlos y en las preguntas abiertas donde la mayoría se queja de la falta de oportunidades producto de una priorización hacia la EC de los médicos. Lo anterior implica que el Estado cae en un incumplimiento directo con la obligación de ofrecer capacitación (desarrollo profesional) a sus funcionarios e indirectamente en la de entregar una atención de calidad a los usuarios del sistema público por medio de profesionales debidamente capacitados, como es obligación en otros países para poder ejercer $^{6,7}$.

Si bien en la actualidad el acceso y las formas de capacitación se han facilitado a través de metodologías e-learning ${ }^{1-3,6-10}$ y el Minsal ha desarrollado programas especiales en este aspecto $^{10}$, no se ve la inclusión (en términos de desarrollo profesional) del componente salud bucal, por lo que políticas como los GES odontológicos pierden un sentido de integralidad, lo que también se ha planteado para el Sistema Chile Crece Contigo ${ }^{12}$.

La percepción de los cursos tomados por los DGZ a través del PAC mejora ostensiblemente, en comparación a la información y oportunidades de acceso. Aún así la mayoría de los DGZ los consideran de mala calidad o no pertinentes para la práctica clínica diaria, mencionado en algunas de las respuestas abiertas, situación que ha sido analizado en otras realidades ${ }^{13,14}$ y plantean la necesidad de desarrollar diagnósticos locales continuos con el fin de ofrecer capacitación, incluso a nivel 
de pregrado ${ }^{14,15}$, que se traduzca en una mejora real de la calidad de atención, más que solo "capacitar por cumplir". Preocupa, además, las reducidas oportunidades para tomar cursos a nivel de diplomado, que brindan mejores posibilidades de desarrollar destrezas y habilidades que los cursos de actualización, pero -sin duda- lo más alarmante es que la mitad de los DGZ apenas recibe una capacitación anual y 1 de cada 4 no recibe ninguna.

El área de administración es la única que pobremente sobresale en los cursos ofrecidos, lo que de acuerdo a las respuestas abiertas se relaciona con la implementación del programa Auge. Sin embargo, sólo 1 de cada 3 DGZ ha accedido a estos cursos. Sorprende comparar lo anterior con el autodiagnóstico de capacidades, que muestran altos porcentajes de dominio, exceptuando en OBE, lo que implicaría:

Un desarrollo de dichas capacidades a nivel de pregrado

y/o por medio de capacitaciones autofinanciadas

y/o un elevado nivel de complacencia en el autodiagnóstico

y/o un inadecuado punto de comparación para la autoevaluación

y/o, finalmente, la posibilidad, mencionada en las respuestas abiertas, que la práctica diaria lleva a un autodesarrollo de estas capacidades, por ejemplo en la atención de niños, preescolares y adultos mayores, producto de las metas sanitarias asociadas a estos grupos.

Lo último exige una profundización investigativa para determinar realmente con qué capacidades cuentan los DGZ (y el resto de los dentistas del sector público) para atender las demandas de los usuarios, más aún a la luz de los preocupantes resultados en capacitación que arroja este estudio.

Un punto aparte lo constituye la OBE, un área básica para el desarrollo de buenas prácticas, que muestra los peores resultados de todas las temáticas consultadas, en línea con otras cifras en el extranjero ${ }^{3}$, donde existe mucho por avanzar en la oferta de cursos por parte de las universidades y, en lo posible, por el Minsal y/o SS, asumiendo su rol asegurador en estos aspectos como ocurre en otros países ${ }^{6,7}$.

La mitad de los DGZ no considera a la EDF como una buena opción para el desarrollo integral de la profesión y la adquisición de las habilidades para entrar a la especialidad (en comparación a los becados autofinanciados), pero la consideran como una buena forma de obtener una beca y la recomendarían a los colegas recién egresados. Lo anterior se resume en un ideario colectivo que considera al DGZ como un sacrificio de aislamiento y atrofia profesional que es recompensado con una beca de especialidad, situación ratificada por los resultados del presente estudio que necesita y puede mejorar por la vía de la EC para lograr un desarrollo profesional de calidad que redunde en la atención de los pacientes.

\section{REFERENCIAS}

1. ARGUEDAS A, CARRILLO M, FRANCESCHI T. Análisis de las líneas y estrategias de formación permanente que utilizan los odontólogos generales, graduados entre los años 1980 y 1990 incorporados al Colegio de Cirujanos Dentistas de Costa Rica. Revista Científica Odontológica CCDCR 2005; 1(1): 4-7.

2. CHRISTENSEN G. Dealing with the increasing need for continuing education. JADA 2007; 138(3): 387-390.

3. NIERI M, MAURO S. Continuing professional development of dental practitioners in prato, Italy. $\mathrm{J}$ Dent Ed 2008; 72(5): 616-625.

4. Ley 19378. Estatutos de Atención Primaria. Diario Oficial de la República de Chile, Santiago, Chile. 13 de abril de 1995.

5. Ley 19.664 "Establece normas especiales para profesionales funcionarios que indica de los servicios de salud y modifica la ley $\mathrm{N}^{\mathrm{o}} 15.076$ ". Diario Oficial de la República de Chile. Santiago, Chile. 28 de enero del 2000.

6. HOPCRAFT M, MANTON D, CHONG P, KO G,ONG P, SRIBALACHANDRAN S, WANG C, YONG P, LIAN L. Participation in continuing professional development by dental practitioners in Victoria, Australia in 2007. Eur J Dent Educ 2010; 14: 227-234.

7. FIRMSTONE V, BULLOCK A, JACKSON D, MANNING R, DAVIES-SLOWIK J, FRAME J. Using evaluation to enhace educational support for dental teams in the UK. J Dent Educ 2010; 74(8):892-901. 
8. ANDREWS K, DEMPS E. Distance Education in the U.S. and Canadian Undergraduate Dental Curriculum. J Dent Educ 2003; 67(4): 427-438.

9. ARGUEDAS A, FRANCESCHINI T, CARRILLO M. Educación en odontología por medios tecnológicos. Revista Científica Odontológica CCDCR 2005; 3(1): 32-40.

10. SHLEYER T, PHAM T. Online continuing dental education. JADA 1999; 130: 848-854.

11. CARABANTES J, GUERRA M, GUILLOU M. Desarrollo de un sistema de educación a distancia en el sector público de salud. Rev Med Chile 2010; 138: 1148-1156.

12. CARTES-VELÁSQUEZ, MARDONES S, PARE-
DES C. Conocimientos y estado de salud bucal en madres beneficiarias del Sistema Chile Crece Contigo. Rev Chil Salud Pública 2009; 13(3): 136-142.

13. GALLIS H. Quality improvement and continuing education in dental education. JADA 2008; 139(6): 656-658.

14. YOUNIES H, BERHAM B, SMITH P. Perception of continuing medical education, professional development, and organizational suppor in the United Arab Emirates. J Contin Educ Health Prof., 2010; 30(4): 251-256.

15. DEPAOLA E, SLAVKIN H. Reforming dental health profession education: A white paper. J Dent Educ 2004; 68(11): 1139-1150.

Usted puede comentar éste y otros artículos publicados en la Revista Chilena de Salud Pública, enviando un correo electrónico a revistasp@med.uchile.cl 